\title{
Endoscope- versus microscope-integrated near-infrared indocyanine green videoangiography in aneurysm surgery
}

\author{
Gerrit Fischer, MD, Jana Rediker, MD, and Joachim Oertel, MD \\ Department of Neurosurgery, Saarland University Medical Center, Homburg/Saar, Germany
}

OBJECTIVE The quality of surgical treatment of intracranial aneurysms is determined by complete aneurysm occlusion while preserving blood flow in the parent, branching, and perforating arteries. For a few years, there has been a nearly noninvasive and cost-effective technique for intraoperative flow evaluation: microscope-integrated indocyanine green videoangiography (mICG-VA). This method allows for real-time information about blood flow in the aneurysm and the involved vessels, but its limitations are seen in the evaluation of structures located in the depth of the surgical field, especially through small craniotomies. To compensate for these drawbacks, an endoscope-integrated ICG-VA (elCG-VA) was developed. The objective of the present study was to assess the use of elCG-VA in comparison with mICG-VA for intraoperative blood flow evaluation.

METHODS In the period between January 2011 and January 2015, 216 patients with a total of 248 intracranial saccular aneurysms were surgically treated in the Department of Neurosurgery of Saarland University Medical Center in Homburg/Saar, Germany. During 95 surgeries in 88 patients with a total of 108 aneurysms, intraoperative evaluation was performed with both elCG-VA and mICG-VA. After clipping, evaluation of complete aneurysm occlusion and flow in the parent, branching, and perforating arteries was performed using both methods. Intraoperative applicability of each technique was compared with the other and with postoperative digital subtraction angiography as a standard evaluation technique.

RESULTS Evaluation of completeness of aneurysm occlusion and of flow in the parent, branching, and perforating arteries was more successful with elCG-VA than with mICG-VA, especially for aneurysm neck assessment (88.9\% vs $69.4 \%)$. For $63.9 \%$ of the aneurysms ( $n=69$ ), both methods were equivalent, but in $30.6 \%$ of the cases $(n=33)$, the elCG-VA provided better results for evaluating the post-clipping situation. In $4.6 \%$ of these aneurysms $(n=5)$, the information given by the additional endoscope considerably changed the surgical procedure. Thus, one residual aneurysm ( $0.9 \%)$, two neck remnants $(1.9 \%)$, and two branch occlusions $(1.9 \%)$ could be prevented. Nevertheless, two incomplete aneurysm occlusions $(1.9 \%)$ and six neck remnants $(5.6 \%)$ were revealed by postoperative digital subtraction angiography.

CONCLUSIONS Endoscope-integrated ICG-VA seems to be an improvement that might increase the quality of aneurysm surgery by providing additional information. It offers higher illumination, magnification, and an extended viewing angle. Its main advantage is its ability to assess deep-seated aneurysms, especially through small craniotomies, but further studies are required.

https://thejns.org/doi/abs/10.3171/2018.4.JNS172650

KEYWORDS indocyanine green videoangiography; aneurysm clipping; endoscope-assisted microsurgery; neuroendoscopy; aneurysm surgery; vascular disorders

$\mathrm{F}$ OR intracranial aneurysms, surgical clipping remains the most definitive treatment strategy. $9,16,18,29,38$ The quality of treatment is decisively determined by complete occlusion of the aneurysm and its neck while maintaining unimpaired blood flow in the parent, branching, and perforating arteries. Postoperative digi- tal subtraction angiography (DSA) is the gold standard for evaluating the anatomical clipping results and sometimes reveals unexpected findings, such as incomplete aneurysm occlusion in $4 \%-19 \%^{11,12,25,27,36,37}$ or accidental parent artery occlusion in $0.3 \%-12 \% .{ }^{25,27,36}$ To keep surgical clipping a competitive alternative to interventional

ABBREVIATIONS AChOA = anterior choroidal artery; $\mathrm{ACOA}=$ anterior communicating artery; $\mathrm{BA}=$ basilar artery; $\mathrm{DSA}=$ digital subtraction angiography; elCG-VA = endoscope-integrated ICG-VA; H\&H = Hunt and Hess; ICA = internal carotid artery; ICG-VA = indocyanine green videoangiography; MCA = middle cerebral artery; mICG-VA = microscope-integrated ICG-VA; SAH = subarachnoid hemorrhage.

SUBMITTED October 20, 2017. ACCEPTED April 23, 2018.

INCLUDE WHEN CITING Published online November 2, 2018; DOI: 10.3171/2018.4.JNS172650. 
treatment, diminishing these adverse results seems to be important.

For direct intraoperative assessment of the aneurysm and the involved vessel structures, microscope-integrated near-infrared indocyanine green videoangiography (mICG-VA) and microvascular Doppler sonography are established techniques. 1,3,8,13,15,20,33-35 Although these techniques provide real-time information about arterial flow, they are still limited to a straight line of view. Information from behind the aneurysm or an angled view of the vessels can only be obtained by applying a micromirror or an endoscope. Particularly, currently available high-quality rigid endoscopes provide excellent visualization of the anatomical structures. . $^{17,19,21,24,31,32}$

The combination of these different imaging modalities underscores the need for an improvement in the quality of surgical aneurysm treatment. The next developmental step is the application of endoscopes with integration of indocyanine green videoangiography (eICG-VA) in the daily routine of aneurysm surgery.

The objective of the current retrospective analysis was to assess the use of eICG-VA in comparison with mICGVA for intraoperative blood flow evaluation by comparing one to the other and to postoperative DSA as a standard evaluation technique.

\section{Methods}

This analysis includes all intracranial saccular aneurysms that were surgically treated with the use of the mICG-VA and eICG-VA for assessment of intraoperative blood flow at the Department of Neurosurgery, Saarland University Medical Center, between January 2011 and January 2015. In this period, 216 patients with a total of 248 intracranial aneurysms were surgically treated. During 95 of these surgeries ( $n=108$ aneurysms) in 88 patients, intraoperative evaluation was performed with both mICG-VA and eICG-VA; when mICG-VA was expected to be limited, eICG-VA was used-for example, in cases of deep-seated aneurysms and/or small craniotomies with reduced light conditions in the depth of the surgical field.

\section{Surgical Procedure}

Each individual aneurysm case-regardless of whether it was an incidental finding or led to a subarachnoid hemorrhage $(\mathrm{SAH})$-was discussed with the interventional radiologists from the department of neuroradiology. If surgical clipping was the preferred treatment strategy, two of the authors (J.O. and G.F.) favored, if applicable, one of the following approaches: a supraorbital subfrontal, pterional transfissural, unilateral interhemispheric, retromastoid, or suboccipital medial approach. The surgeries were performed using a standard microsurgical technique. If the surgeon felt that mICG-VA would be insufficient, eICG-VA was additionally performed. Both mICG-VA and eICG-VA were performed after clipping in each of these cases. Special attention was given to the patency of the parent, branching, and perforating arteries and to the degree of aneurysm occlusion. Microvascular Doppler sonography (DWL Electronic Systems Inc.) for the assessment of vessel patency was also used as a matter of routine. Postoperative DSA was performed as a standard evaluation technique. Intraoperative applicability of each ICG-VA technique was compared to the other and to postoperative DSA.

\section{Microscope-Integrated ICG-VA}

Intraoperative mICG-VA was performed using the OPMI Pentero (Carl Zeiss), a commercially available surgical microscope. This technique has been described in detail elsewhere. ${ }^{10,33-35}$ ICG-VA with a standard 0.5-mg/ $\mathrm{kg}$ intravenous bolus of ICG (ICG-PULSION) was performed after clipping of each aneurysm. The real-time angiographic imaging is displayed on a video screen and can be recorded for further analysis during or after surgery.

\section{Endoscope-Integrated ICG-VA}

Intraoperative eICG-VA was performed with a nearinfrared endoscopic system (Karl Storz GmbH). It consists of the D-Light P light source, the Tricam SL II 3-chip CCU camera, and a Tricam PDD 3-chip camera head, which was optimized for the near-infrared endoscopic system. The endoscope itself is a $0^{\circ}$ optic with an outer diameter of $5.8 \mathrm{~mm}$ and shaft length of $19 \mathrm{~cm}$. With a rotary switch, the user can choose between standard white light and ICG mode. ${ }^{6}$ ICG-VA with a standard $0.5-\mathrm{mg} / \mathrm{kg}$ intravenous bolus of ICG (ICG-PULSION) was performed after clipping of each aneurysm. The intravascular fluorescence can be observed on the video screen in real time and recorded for further analysis during or after surgery.

In all cases, mICG-VA was performed before eICGVA, and after a period of 3-4 minutes (which would comply with the half-life of ICG's plasma elimination), eICG-VA was performed with an additional bolus of the fluorescence dye.

\section{Acquisition of Data}

Patient data, as well as pre- and postoperative radiological images, were obtained from the departments' digital patient management program. Assessment of intraoperative findings was performed by video analysis and analysis of the surgical reports.

\section{Results}

A total of 88 patients with 108 aneurysms were surgically treated in 95 sessions. The patient population consisted of 59 women $(67.0 \%)$ and 29 men (33.0\%), whose ages ranged from 30 to 87 years (mean 56.4 years). Eleven patients $(12.5 \%)$ had multiple aneurysms treated in one surgery. Seventeen patients presented with SAH. At the time of admission, 4 of the SAHs were classified as Hunt and Hess $(\mathrm{H} \& \mathrm{H})$ grade $\mathrm{I}, 4$ as $\mathrm{H} \& \mathrm{H}$ grade II, 2 as $\mathrm{H} \& \mathrm{H}$ grade III, and 7 as $\mathrm{H} \& \mathrm{H}$ grade IV. Patient characteristics are listed in Table 1 . In $94.4 \%$ of the cases (102/108), aneurysms were located in the anterior circulation and in $5.6 \%(6 / 108)$ in the posterior circulation. Distribution of the aneurysms is shown in Table 2 . The supraorbital approach was used $47(49.5 \%)$ of the 95 surgeries to treat 52 aneurysms; the pterional approach was applied 41 (43.2\%) of the 95 surgeries to treat 49 aneurysms. Four aneurysms 
TABLE 1. Characteristics of 88 patients

\begin{tabular}{ccc}
\hline Characteristic & No. of Patients & $\%$ of Patients \\
\hline No. of patients & 88 & 100 \\
\hline Female & 59 & 67 \\
\hline Male & 29 & 33 \\
\hline SAH score & 17 & 19.3 \\
\hline H\&H I & 4 & 4.5 \\
\hline H\&H II & 4 & 4.5 \\
\hline H\&H III & 2 & 2.3 \\
\hline H\&H IV & 7 & 8.0 \\
\hline H\&H V & 0 & 0 \\
\hline Multiple aneurysms & 11 & 12.5 \\
\hline 2 & 9 & 10.2 \\
\hline 3 & 2 & 2.3 \\
\hline
\end{tabular}

were clipped via the interhemispheric approach. The retromastoid approach was used twice, and the medial suboccipital approach was used in one case (Table 3). Six of the patients (5.6\%) had undergone prior insufficient aneurysm treatment: 3 patients had insufficiently coiled aneurysms ( 2 anterior communicating artery $[\mathrm{ACoA}]$ and 1 posterior inferior cerebellar artery) and 3 aneurysms (middle cerebral artery [MCA], ACoA, and internal carotid artery [ICA]) were incompletely clipped previously.

\section{Intraoperative eICG-VA and mICG-VA}

Evaluation of the post-clipping status with eICG-VA was possible in 107 of the 108 aneurysms. The other case was an aneurysm of the basilar artery (BA) tip. After clip application, the interpeduncular space was too narrow to bring the endoscope to the BA trunk.

Intraoperative investigation after clip application using mICG-VA was feasible for 104 aneurysms. In the other 4 cases, it was impossible to visualize and evaluate one of the involved vessel structures. Three of these cases were aneurysms of the BA clipped via a pterional approach and 1 case was an aneurysm of the anterior choroidal artery (AChoA) treated via a supraorbital approach. After clip application, the light conditions in the depth of the surgical field were restricted, and the visualization could not be achieved. No side effects were observed. The results of intraoperative assessment are illustrated in Fig. 1 and unexpected findings are summarized in Table 4.

\section{Assessment of Complete Aneurysm Occlusion}

Assessment was possible in 107 of the 108 aneurysms (99.1\%) using eICG-VA and in 102 aneurysms (94.4\%) using mICG-VA. With mICG-VA, 4 incomplete aneurysm occlusions could be detected and intraoperatively corrected $(3.7 \%)$. eICG-VA revealed one more of these unexpected intraoperative incomplete occlusions (4.6\%) in a case of an aneurysm of the MCA that was clipped via a supraorbital approach. Thus, eICG-VA could have prevented an additional residual aneurysm filling, but 2 aneurysm remnants $(1.9 \%)$ were revealed on postoperative DSA and had to be retreated.
TABLE 2. Distribution of aneurysm locations

\begin{tabular}{ccc}
\hline Location of Aneurysm & No. of Aneurysms & \% of Aneurysms \\
\hline MCA & 62 & 57.4 \\
\hline ACoA & 26 & 24.1 \\
\hline ICA & 6 & 5.6 \\
\hline PerA & 4 & 3.7 \\
\hline AChoA & 3 & 2.8 \\
\hline PICA & 3 & 2.8 \\
\hline BA & 3 & 2.8 \\
\hline OphthA & 1 & 0.9 \\
\hline Total & 108 & 100 \\
\hline
\end{tabular}

OphthA = ophthalmic artery; PerA = pericallosal artery; PICA = posterior inferior cerebellar artery.

A case of incomplete aneurysm occlusion detected only on eICG-VA is shown in Fig. 2 and Video 1.

VIDEO 1. A 50-year-old woman with an incidentally detected right MCA bifurcation aneurysm clipped via a right supraorbital approach with intracilian skin incision. Copyright Department of Neurosurgery, Saarland University Medical Center and Saarland University Faculty of Medicine, Homburg/Saar, Germany. Published with permission.

Click here to view.

\section{Evaluation of the Aneurysm Neck}

Aneurysm neck assessment was possible in 75 (69.4\%) of 108 aneurysms using mICG-VA and in $96(88.9 \%)$ using eICG-VA. There were different reasons for the failure of mICG-VA: poor light conditions in the depth of the field after clipping or through small craniotomies (supraorbital approach) and, in cases of deep-seated aneurysms (BA, AChoA, and ICA), hidden vessel structures and a restricted viewing angle of the microscope. mICG-VA revealed 8 neck remnants $(7.4 \%)$ after clipping and eICG-VA revealed $10(9.3 \%)$. Thus, two additional complete aneurysm occlusions could be achieved due to the application of the endoscope. Nevertheless, postoperative DSA showed 6 additional residual aneurysm necks (5.6\%), but none of them had to be retreated at the time of diagnosis and angiographic observation.

\section{Detection of Flow in Parent Vessels}

Detection of flow in parent vessels was possible in 124 of 129 evaluated parent arteries (96.1\%) with mICG-VA. Using eICG-VA, however, parent vessel flow could be detected in 128 of 129 parent arteries $(99.2 \%)$. There was no

\section{TABLE 3. Summary of 95 surgical approaches}

\begin{tabular}{lcc}
\hline \multicolumn{1}{c}{ Approach } & No. of Approaches & \% of Approaches \\
\hline Supraorbital & 47 & 49.5 \\
\hline Pterional & 41 & 43.2 \\
\hline Interhemispheric & 4 & 4.2 \\
\hline Retromastoid & 2 & 2.1 \\
\hline Suboccipital medial & 1 & 1.1 \\
\hline Total & 95 & 100 \\
\hline
\end{tabular}




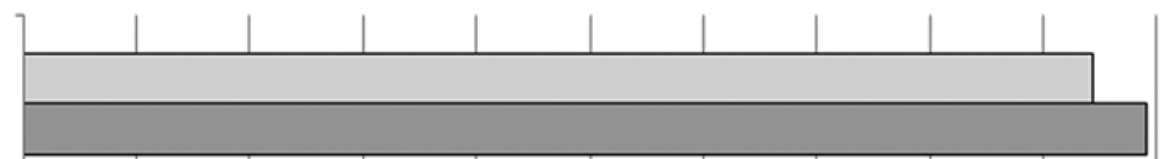

Aneurysm neck assessment
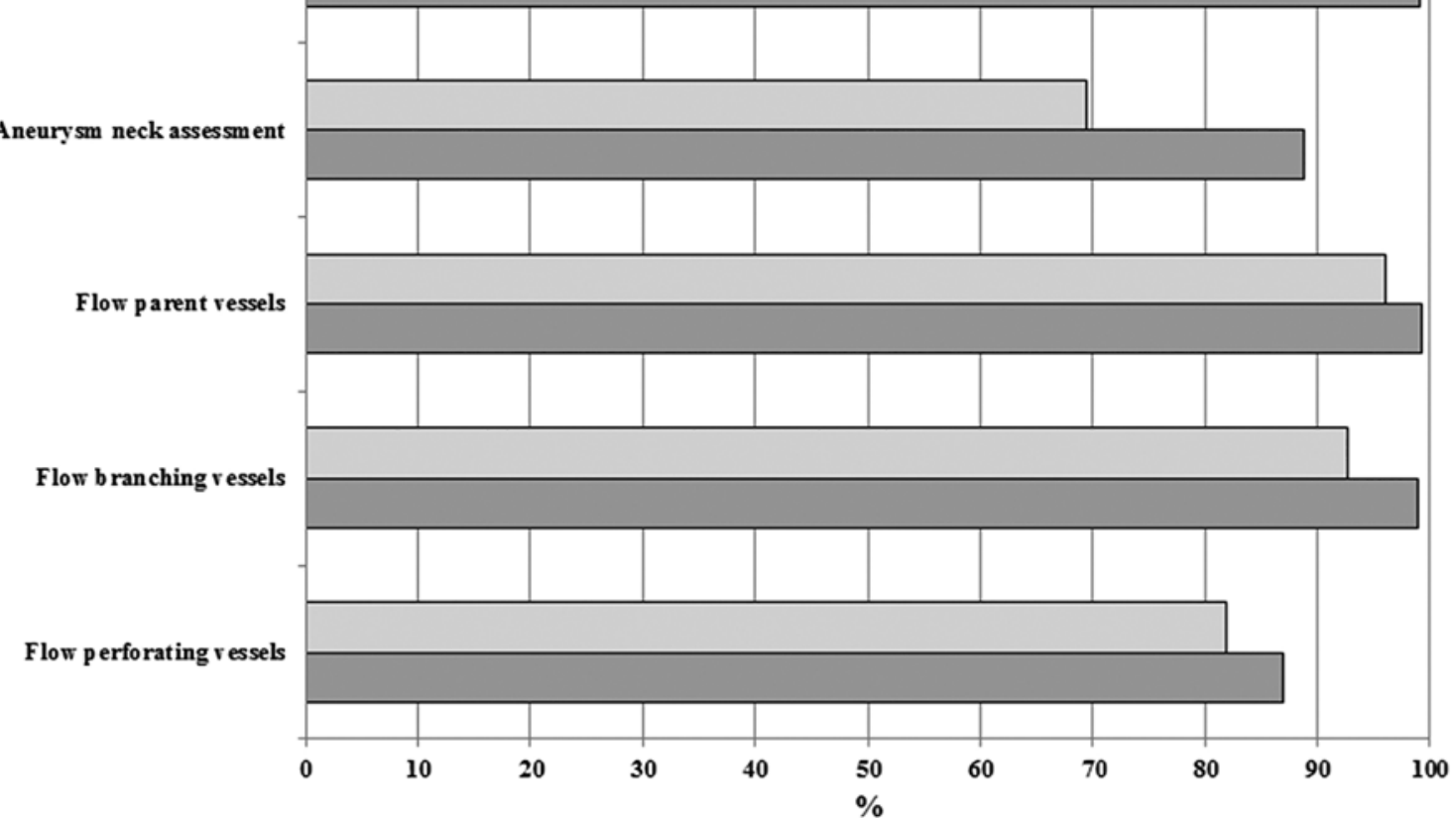

口MICG-VA

口eICG-VA

FIG. 1. Intraoperative results of detecting flow in the vessel sections with mICG-VA and elCG-VA.

detection of accidental occlusion of parent vessels, either with intraoperative mICG-VA/eICG-VA or in postoperative DSA.

\section{Visualization of Flow in Branching Vessels}

Visualization of flow in branching vessels was possible in 191 (92.7\%) of 206 cases with mICG-VA and in 204 (99.0\%) with eICG-VA. A total of 3 accidental branch occlusions were shown by eICG-VA $(2.8 \%)$, one of which was also detected with microscopic mICG-VA $(0.9 \%)$. eICG-VA was additionally used for evaluation after clip application led to an immediate repositioning of the clip. Postoperative DSA demonstrated no additional unexpected vessel compromises. A case of eICG-VA improved parent vessel visualization and assessment is shown in Fig. 3 and Video 2.

VIDEO 2. Incidentally detected, partly thrombosed ACOA aneurysm in a 30-year-old woman in whom clipping was performed via a right supraorbital approach with intracilian skin incision. Copyright Department of Neurosurgery, Saarland University Medical Center

TABLE 4. Detection of unexpected intraoperative and postoperative findings

\begin{tabular}{lccc}
\hline \multirow{2}{*}{ Finding } & \multicolumn{3}{c}{ No. of Findings } \\
\cline { 2 - 4 } & mICG-VA & elCG-VA & Postop DSA \\
\hline Residual aneurysm & 4 & 5 & 2 \\
\hline Neck remnant & 8 & 10 & 6 \\
\hline Branch occlusion & 1 & 3 & 0 \\
\hline Parent vessel occlusion & 0 & 0 & 0 \\
\hline
\end{tabular}

and Saarland University Faculty of Medicine, Homburg/Saar, Germany. Published with permission. Click here to view.

\section{Visualization of Perforating Arteries}

A total of 61 perforating arteries were visualized on DSA, 50 of which could be accurately assessed with mICG-VA (82.0\%) and 53 with eICG-VA (86.9\%). No accidental compromise of perforating arteries was detected by intraoperative mICG-VA or eICG-VA, but postoperative CT scanning revealed small ischemic infarctions in areas supplied by perforating arteries in 7 (6.5\%) of 108 aneurysms. A case of postoperative ischemic infarction due to a perforator occlusion is shown in Fig. 4.

\section{Comparison of Both Methods}

In $63.9 \%$ of the cases $(n=69)$, mICG-VA and eICG-VA were equivalent in terms of the accuracy with which they demonstrated intraoperative complete aneurysm occlusion and flow in parent, branching, and perforating arteries. However, in $30.6 \%(n=33)$, eICG-VA provided better results with which to evaluate the post-clipping picture (Table 5). In 28 of these cases, eICG-VA delivered better images with higher resolution, illumination, and magnification. In 5 of these cases, the information given by intraoperative eICG-VA significantly changed the surgical procedure: 1 residual aneurysm $(0.9 \%), 2$ neck remnants (1.9\%), and 2 branch occlusions (1.9\%) could only be detected by using eICG-VA. Thus, these lesions could be prevented by the additional use of this new intraoperative imaging technique and by intermediate repositioning of the applied clip or by immediate positioning of an addi- 


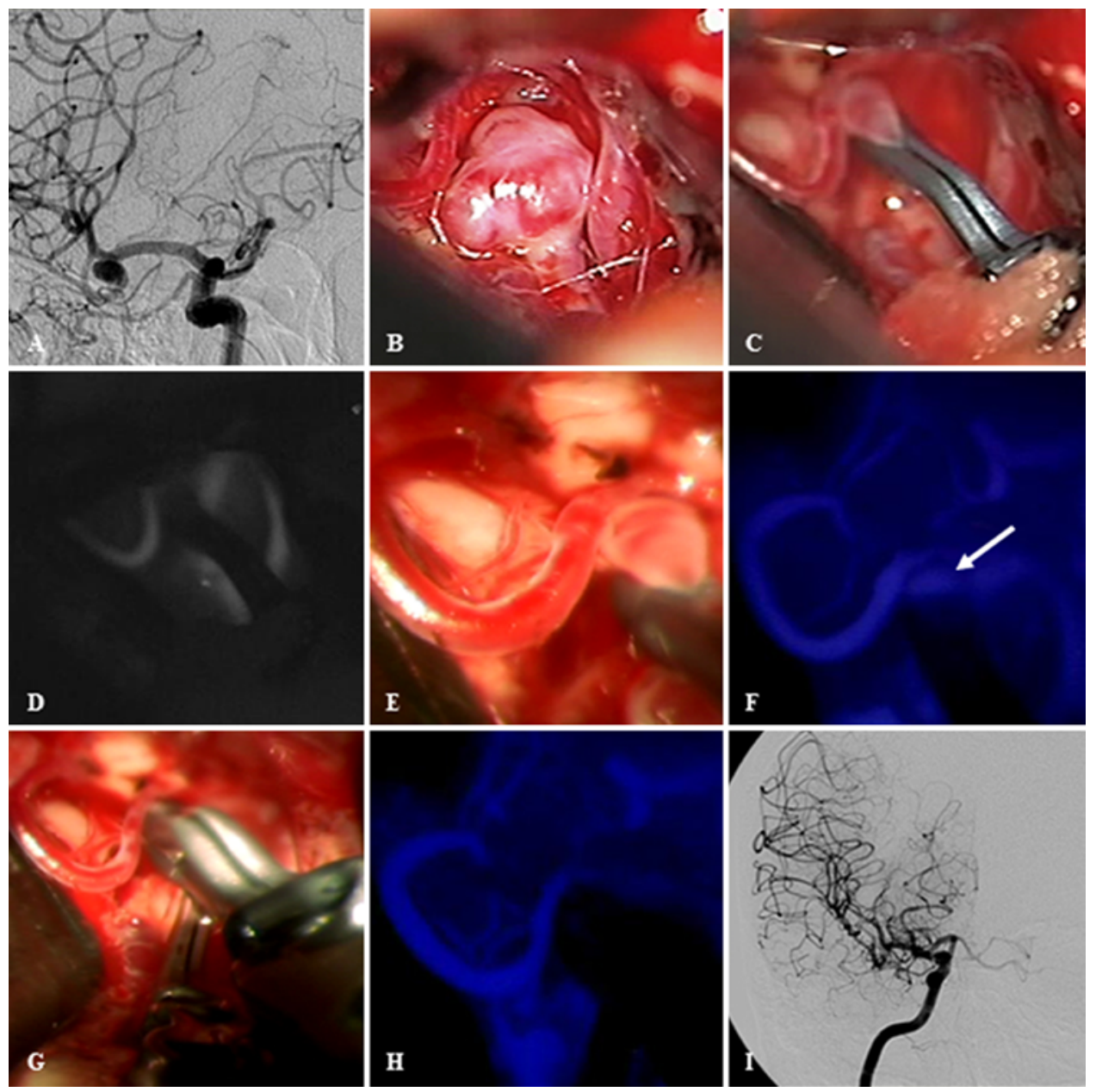

FIG. 2. Studies in a 50-year-old woman with an incidentally detected right MCA bifurcation aneurysm clipped via a right supraorbital approach with intracilian skin incision. A: Preoperative DSA image showing the aneurysm located at the bifurcation of the MCA. B: Microscopic view prior to clipping. C: Microscopic view after clipping. D: mICG-VA view after clipping showing no fluorescence inside the aneurysm; the aneurysm seems to be completely occluded. E: Endoscopic view after clipping. F: elCG-VA view after clipping revealing incompletely occluded aneurysm with residual fluorescence inside (arrow). An additional clip was applied. G: Endoscopic view after additional clip application. H: elCG-VA view after additional clip application showing absence of fluorescence inside the aneurysm. I: Postoperative DSA image confirming complete aneurysm occlusion without any vessel compromise. Figure is available in color online only.

tional clip. mICG-VA was found to be superior in 5 cases (4.6\%) because of its improved imaging quality. In 1 case $(0.9 \%)$, both modalities failed.

\section{Discussion}

Microsurgical clipping of intracranial aneurysms aims for complete aneurysm occlusion while preserving unimpaired blood flow in the parent, branching, and perforating arteries. Even at the most experienced centers, however, hemodynamically relevant constrictions or vessel occlusions, as well as incomplete aneurysm occlusion, can be revealed by postoperative DSA. For example, review of the literature shows postoperative aneurysm remnants to be reported in $4 \%-19 \%$ of cases ${ }^{11,25,27,29,36,38}$ and accidental parent artery occlusion in 0.3\%-12\%. ${ }^{25,27,36}$ Many authors regard DSA to be the ideal evaluation modality., $7,22,23,25,26,39$ For several reasons, however, it has failed to become routinely used during intracranial aneurysm surgeries. Normally, the quality of clip placement is evaluated by direct visual inspection. Nowadays, there are additional and well-established techniques for intraoperative visual clip assessment: mICG-VA and endoscopic inspection. mICG-VA is a less invasive, safe, and easy method that provides real-time information about the blood flow in the involved vessel structures and a good evaluation of complete aneurysm occlusion. ${ }^{8,20,33-35}$ However, these benefits apply only to structures within the viewing angle of the surgical approach. Thus, the visual field is frequently restricted to the field of the direct line of sight under microscopic view., $4,8,15$ Careful retraction and microsurgical dissection of the aneurysm may help by providing additional visual information from behind the aneurysm or hidden corners. However, intraoperative rupture may occur even as a result of gentle aneurysm or vessel retraction and overexposure of the aneurysm. 

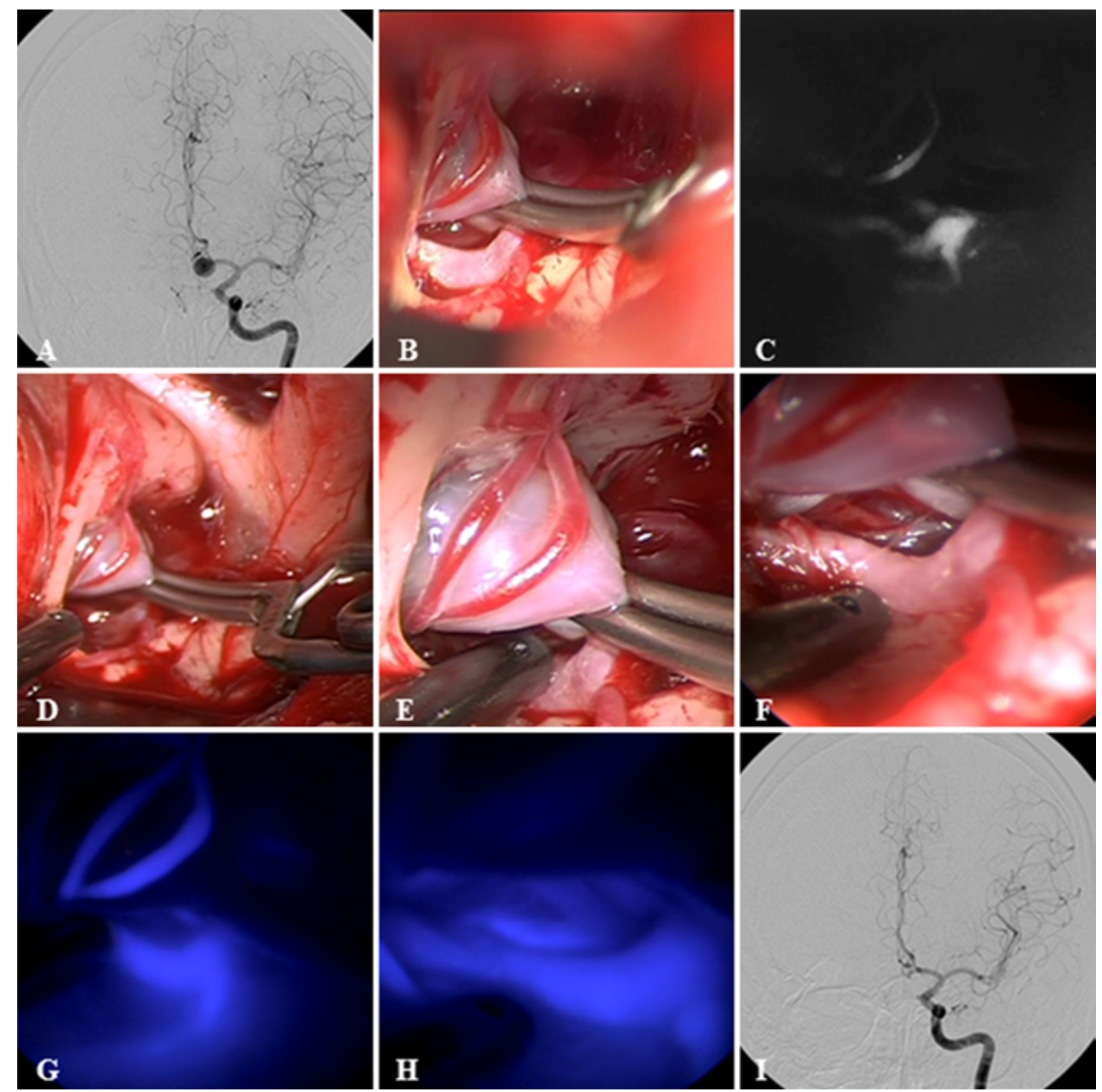

FIG. 3. Incidentally detected, partly thrombosed ACoA aneurysm in a 30-year-old woman in whom clipping was performed via a right supraorbital approach with intracilian skin incision. A: Preoperative DSA image showing the ACoA aneurysm. B: Microscopic view of the aneurysm after clipping revealing optic control only over the proximal contralateral $\mathrm{A}_{2}$ segment. The distal part cannot be visualized because the large, partly thrombosed aneurysm sac is hardly retractable. C: mICG-VA image after clipping provides no additional information. D and E: Endoscopic views over the operative field after clipping. F: Close-up endoscopic view where the distal part of the contralateral $A_{2}$ segment can be visualized. The part in between is hidden by the rigid, partly thrombosed aneurysm sac. G and H: elCG-VA images after clipping. With elCG-VA, flow can be assured in the distal part of the contralateral $\mathrm{A}_{2}$ segment. I: Postoperative DSA confirming complete aneurysm occlusion without any vessel compromise. Figure is available in color online only.

Endoscope-assisted microsurgery has some benefits of its own: it provides a higher illumination in the depth of the surgical field, offers an extended viewing angle, and provides detailed pathoanatomical information in a close-up fashion. The additional endoscopic enhancement of the visual field before, during, and after microsurgical aneurysm occlusion has proven to be safe and effective, and it increases the quality of treatment, although only plain anatomical details can be evaluated. $17,19,21,24,31,32$ The logical desire to combine the benefits of the two procedures is realized through eICG-VA. The initial experiences with eICG-VA in aneurysm surgery were reported by Bruneau et al. and Nishiyama et al. in 1 and 3 cases, respectively, of surgically treated unruptured aneurysms. ${ }^{6,30}$ The first study to evaluate the usefulness of eICG-VA in comparison to mICG-VA was published by Mielke et al. and included 30 unruptured and ruptured aneurysms. ${ }^{28}$ The authors concluded that eICG-VA was a useful modality for intraoperative assessment during cerebral aneurysm surgery. Its main advantage is seen in its ability to provide real-time information about vessel structures hidden from the microscopic view.

The goal of the current series was to assess the intraoperative applicability of eICG-VA in comparison to mICGVA. A total of 108 unruptured and ruptured aneurysms are included. This series has the highest number of aneurysms to be reported on and represents the first report concerning application of eICG-VA to aneurysms of the posterior circulation. Other important new features are the evaluation of eICG-VA through keyhole craniotomies and the comparison of intraoperative ICG-VA results to postoperative DSA findings. The following aspects were reviewed: completeness of aneurysm occlusion, neck remnants, and parent, branching, and perforator artery occlusion. Compared 


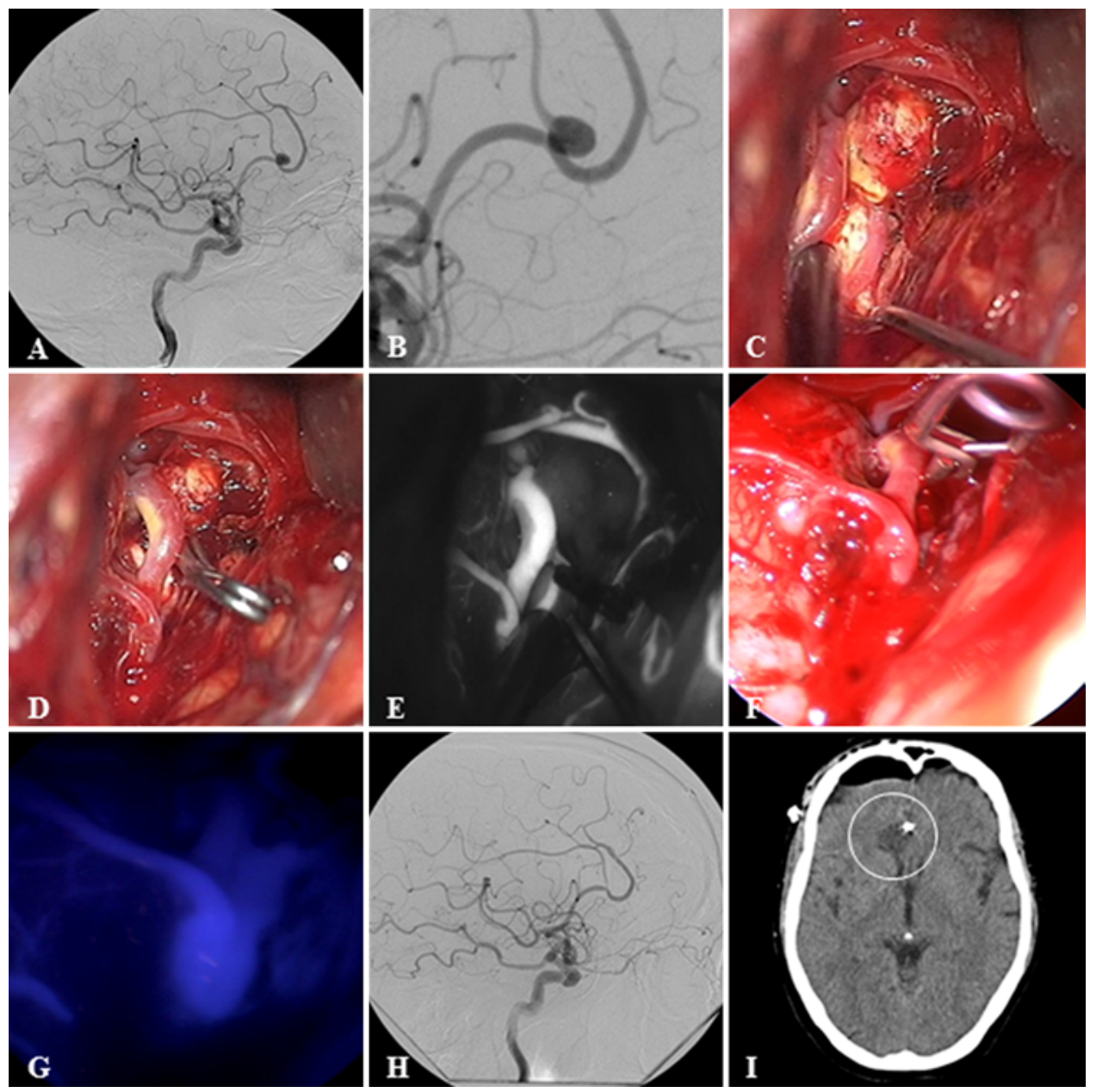

FIG. 4. Studies in a 71-year-old woman with an incidentally detected calcified aneurysm of the right pericallosal artery treated via an interhemispheric approach. A and B: Preoperative DSA images showing the aneurysm. C and D: Microscopic views prior to (C) and after (D) clip application. E: mICG-VA image obtained after clipping showing the aneurysm completely occluded and without any vessel compromise. F: Endoscopic inspection after clipping allows a close-up view and a different viewing angle. G: elCG-VA after clipping provides no additional information. H: Postoperative DSA image confirming complete aneurysm occlusion without any vessel compromise. I: Postoperative cranial CT scan revealing a small ischemic infarction (circle) in an area supplied by a perforating artery. Intraoperatively, neither mICG-VA nor elCG-VA revealed this perforator. Figure is available in color online only.

with DSA, the main advantages of eICG-VA and mICGVA are that these modalities are less invasive, easier to apply, and have relatively low costs.

In the present series, a clear fluorescent signal could be obtained in nearly all cases. However, the endoscope available for this assessment is rather large, and handling it was more demanding than what is required when using a standard endoscope. For this reason, the insertion of the endoscope cannot be controlled under a microscopic view. Further dissection and aneurysm clipping or clip repositioning was always performed under microscopic view. Additionally, the fluorescent signal was not as bright as with the microscope, and the operating theater had to be darkened to allow for obtaining good quality images. Investigating deep-seated aneurysms such as those of the BA was possible. However, although the tip of the endoscope could be brought in close proximity to the target area, evaluation of the vessels remained difficult because the diameter of the endoscope prohibited the introduction of the scope deep in the surgical field.

Nevertheless, after navigation of all these difficulties, eICG-VA provided very useful angiographic images. Because of the wide angle of view, more details could be visualized than were seen with the microscope. In selected cases, even additional information on vessels behind the aneurysm could be obtained. Complications in direct relation to the endoscope or use of ICG were not observed in this series. Both methods compete with each other to a certain degree for intraoperative evaluation, but nevertheless, 2 aneurysm remnants $(1.9 \%)$ and 6 neck remnants $(5.6 \%)$ were revealed by postoperative DSA. This underlines the fact that neither method is infallible. During mICG-VA, the viewing angle is limited by the craniotomy. eICG-VA and endoscopic inspection with angled optics can extend the viewing angle, but a $360^{\circ}$ view cannot be achieved. Although both methods 
TABLE 5. Summary of 33 cases in which eICG-VA provided better results than mICG-VA

\begin{tabular}{|c|c|c|c|c|c|c|}
\hline $\begin{array}{l}\text { Case } \\
\text { No. }\end{array}$ & Sex & $\begin{array}{l}\text { Age } \\
\text { (yrs) }\end{array}$ & $\begin{array}{l}\text { Aneurysm } \\
\text { Location }\end{array}$ & Approach & Comment & Consequence \\
\hline 1 & M & 48 & MCA & SO & Neck remnant only detected w/ elCG-VA & Additional clip \\
\hline 2 & $\mathrm{~F}$ & 30 & $\mathrm{ACoA}$ & SO & Flow in contralat $A_{2}$ only accurately assessed w/ elCG-VA (Fig. 3, Video 2) & Higher quality \& safety \\
\hline 3 & $\mathrm{~F}$ & 65 & MCA & SO & Higher-resolution \& more-detailed images provided by elCG-VA & Higher quality \& safety \\
\hline 4 & $\mathrm{~F}$ & 50 & MCA & SO & Residual aneurysm filling only detected w/ elCG-VA (Fig. 2, Video 1) & Additional clip \\
\hline 5 & $\mathrm{~F}$ & 61 & MCA & Pter & Branch occlusion only detected w/ elCG-VA & Clip reposition \\
\hline 6 & M & 62 & $\mathrm{ACoA}$ & SO & Flow in contralat $A_{1}, A_{2}$, \& neck only accurately assessed w/ elCG-VA & Higher quality \& safety \\
\hline 7 & $\mathrm{~F}$ & 46 & MCA & Pter & More-detailed images provided by elCG-VA & Higher quality \& safety \\
\hline 8 & $\mathrm{~F}$ & 47 & $\mathrm{ACoA}$ & SO & More-detailed images provided by elCG-VA & Higher quality \& safety \\
\hline 9 & $\mathrm{~F}$ & 48 & AChoA & SO & mICG-VA failed; accurate assessment only possible w/ ICG-VA & Higher quality \& safety \\
\hline 10 & M & 44 & AChoA & SO & Neck assessment only sufficient w/ elCG-VA & Higher quality \& safety \\
\hline 11 & M & 59 & $\mathrm{ACoA}$ & SO & Branch occlusion only detected w/ elCG-VA & Clip reposition \\
\hline 12 & M & 60 & MCA & Pter & Perforator only visualized by elCG-VA & Higher quality \& safety \\
\hline 13 & $\mathrm{~F}$ & 63 & BA & Pter & $\begin{array}{l}\text { After clip application, mICG failed; assessment w/ elCG-VA was limited } \\
\text { but possible }\end{array}$ & Higher quality \& safety \\
\hline 14 & $\mathrm{~F}$ & 30 & AChoA & Pter & Neck, branch, \& perforator only visualized by elCG-VA & Higher quality \& safety \\
\hline 15 & $\mathrm{~F}$ & 73 & $\mathrm{ACoA}$ & SO & $\begin{array}{l}\text { Better light conditions provided by elCG-VA; both methods failed for neck } \\
\text { assessment }\end{array}$ & $\begin{array}{l}\text { Higher quality \& safety } \\
\text { but limited }\end{array}$ \\
\hline 16 & $\mathrm{~F}$ & 51 & MCA & SO & Neck assessment only successful w/ elCG-VA & Higher quality \& safety \\
\hline 17 & $\mathrm{~F}$ & 52 & MCA & SO & $\mathrm{M}_{2}$ assessment only successful w/ elCG-VA & Higher quality \& safety \\
\hline 18 & M & 53 & MCA & SO & Neck assessment only successful w/ elCG-VA & Higher quality \& safety \\
\hline 19 & M & 73 & $\mathrm{ACoA}$ & SO & Contralat $\mathrm{A}_{2}$ \& neck only visualized by ICG-VA & Higher quality \& safety \\
\hline 20 & $\mathrm{~F}$ & 52 & BA & Pter & mICG-VA failed; elCG-VA successful & Higher quality \& safety \\
\hline 21 & $\mathrm{~F}$ & 52 & MCA & Pter & $\begin{array}{l}\text { Both methods failed for perforator assessment; } 1 \text { branch only visualized } \\
\text { by elCG-VA }\end{array}$ & Higher quality \& safety \\
\hline 22 & $\mathrm{~F}$ & 67 & MCA & SO & Better neck visualization w/ elCG-VA & Higher quality \& safety \\
\hline 23 & M & 31 & $\mathrm{ACoA}$ & SO & Neck remnant only detected w/ elCG-VA & Clip reposition \\
\hline 24 & M & 50 & MCA & Pter & Neck assessment only sufficient w/ elCG-VA & Higher quality \& safety \\
\hline 25 & M & 51 & MCA & Pter & Neck assessment only sufficient w/ elCG-VA & Higher quality \& safety \\
\hline 26 & $\mathrm{~F}$ & 49 & ICA & Pter & Neck assessment only sufficient w/ elCG-VA & Higher quality \& safety \\
\hline 27 & $\mathrm{~F}$ & 63 & ACoA & SO & Assessment of complete aneurysm occlusion only sufficient w/ elCG-VA & Higher quality \& safety \\
\hline 28 & M & 51 & MCA & SO & Neck assessment only sufficient w/ elCG-VA & Higher quality \& safety \\
\hline 29 & M & 59 & $\mathrm{ACoA}$ & Pter & More-detailed images provided by elCG-VA & Higher quality \& safety \\
\hline 30 & $\mathrm{~F}$ & 39 & $\mathrm{ACoA}$ & SO & $\begin{array}{l}\text { Assessment of branching arteries only visualized by elCG-VA but higher } \\
\text { resolution w/ mICG-VA }\end{array}$ & Higher quality \& safety \\
\hline 31 & $\mathrm{~F}$ & 62 & MCA & Pter & Neck assessment only sufficient w/ elCG-VA & Higher quality \& safety \\
\hline 32 & $\mathrm{~F}$ & 62 & MCA & Pter & Higher-resolution \& more-detailed images provided by elCG-VA & Higher quality \& safety \\
\hline 33 & $\mathrm{~F}$ & 53 & ICA & Pter & Neck assessment only sufficient w/ elCG-VA & Higher quality \& safety \\
\hline
\end{tabular}

Pter $=$ pterional transfissural approach; $\mathrm{SO}=$ supraorbital subfrontal approach .

are called "angiography," they are not exactly comparable with 3D rotational DSA.

Disadvantages of the eICG-VA might be the limited availability of endoscopic devices and the need for specially trained staff in the operating room any time they are used. Additionally, a profound knowledge of specific anatomical features under endoscopic view combined with trained hand-eye coordination is essential. The fact that the endoscope's camera can only detect the fluorescent signal in a dark environment is also seen as a drawback.
The most significant disadvantage is the large diameter and length of the endoscope.

\section{Limitations}

One limitation of our series is the fact that not all cases of aneurysm clipping performed during the study period were included. Due to the large size of the endoscope, application was limited to very select cases, since handling was inconvenient. eICG-VA was only additionally performed in cases in which mICG-VA was expected to 
be limited-e.g., in cases of deep-seated aneurysms and/ or small craniotomies with reduced light conditions in the depth of the surgical field. Thus, there is a selection of the cases before evaluation of the intraoperative situation. Another weak point is that the cases were analyzed retrospectively.

\section{Conclusions}

We believe that the benefits derived from endoscopic ICG-VA combined with the benefits of a well-established procedure like mICG-VA might increase the quality of aneurysm treatment even more. However, both techniques also have specific drawbacks in comparison with DSA that could be compensated for by each other, to a certain extent. Endoscopic and microscopic ICG-VA should be considered as complementary but not competitive.

Although the use of both modalities could reduce unexpected findings such as incomplete aneurysm occlusion or accidental vessel occlusion, total prevention could not be achieved. In selected cases, intraoperative DSA might still be indicated. Further studies will be required to draw definitive consequences.

\section{References}

1. Amin-Hanjani S, Meglio G, Gatto R, Bauer A, Charbel FT: The utility of intraoperative blood flow measurement during aneurysm surgery using an ultrasonic perivascular flow probe. Neurosurgery 58 (4 Suppl 2):ONS-305-ONS-312, 2006

2. Bailes JE, Deeb ZL, Wilson JA, Jungreis CA, Horton JA: Intraoperative angiography and temporary balloon occlusion of the basilar artery as an adjunct to surgical clipping: technical note. Neurosurgery 30:949-953, 1992

3. Bailes JE, Tantuwaya LS, Fukushima T, Schurman GW, Davis D: Intraoperative microvascular Doppler sonography in aneurysm surgery. Neurosurgery 40:965-972, 1997

4. Balamurugan S, Agrawal A, Kato Y, Sano H: Intra operative indocyanine green video-angiography in cerebrovascular surgery: an overview with review of literature. Asian J Neurosurg 6:88-93, 2011

5. Barrow DL, Boyer KL, Joseph GJ: Intraoperative angiography in the management of neurovascular disorders. Neurosurgery 30:153-159, 1992

6. Bruneau M, Appelboom G, Rynkowski M, Van Cutsem N, Mine B, De Witte O: Endoscope-integrated ICG technology: first application during intracranial aneurysm surgery. Neurosurg Rev 36:77-85, 2013

7. Chiang VL, Gailloud P, Murphy KJ, Rigamonti D, Tamargo RJ: Routine intraoperative angiography during aneurysm surgery. J Neurosurg 96:988-992, 2002

8. Dashti R, Laakso A, Niemelä M, Porras M, Hernesniemi $\mathrm{J}$ : Microscope-integrated near-infrared indocyanine green videoangiography during surgery of intracranial aneurysms: the Helsinki experience. Surg Neurol 71:543-550, 2009

9. David CA, Vishteh AG, Spetzler RF, Lemole M, Lawton MT, Partovi S: Late angiographic follow-up review of surgically treated aneurysms. J Neurosurg 91:396-401, 1999

10. de Oliveira JG, Beck J, Seifert V, Teixeira MJ, Raabe A: Assessment of flow in perforating arteries during intracranial aneurysm surgery using intraoperative near-infrared indocyanine green videoangiography. Neurosurgery 61 (3 Suppl):63-73, 2007

11. Drake CG, Allcock JM: Postoperative angiography and the "slipped" clip. J Neurosurg 39:683-689, 1973

12. Feuerberg I, Lindquist C, Lindqvist M, Steiner L: Natural history of postoperative aneurysm rests. J Neurosurg 66:30 34, 1987

13. Firsching R, Synowitz HJ, Hanebeck J: Practicability of intraoperative microvascular Doppler sonography in aneurysm surgery. Minim Invasive Neurosurg 43:144-148, 2000

14. Fischer G, Oertel J, Perneczky A: Endoscopy in aneurysm surgery. Neurosurgery 70 (2 Suppl Operative):184-191, 2012

15. Fischer G, Stadie A, Oertel JM: Near-infrared indocyanine green videoangiography versus microvascular Doppler sonography in aneurysm surgery. Acta Neurochir (Wien) 152:1519-1525, 2010

16. Fogelholm R, Hernesniemi J, Vapalahti M: Impact of early surgery on outcome after aneurysmal subarachnoid hemorrhage. A population-based study. Stroke 24:16491654,1993

17. Fries G, Perneczky A: Endoscope-assisted brain surgery: part 2-analysis of 380 procedures. Neurosurgery 42:226-232, 1998

18. Hernesniemi J, Vapalahti M, Niskanen M, Tapaninaho A, Kari A, Luukkonen M, et al: One-year outcome in early aneurysm surgery: a 14 years experience. Acta Neurochir (Wien) 122:1-10, 1993

19. Hopf NJ, Perneczky A: Endoscopic neurosurgery and endoscope-assisted microneurosurgery for the treatment of intracranial cysts. Neurosurgery 43:1330-1337, 1998

20. Imizu S, Kato Y, Sangli A, Oguri D, Sano H: Assessment of incomplete clipping of aneurysms intraoperatively by a nearinfrared indocyanine green-video angiography (Niicg-Va) integrated microscope. Minim Invasive Neurosurg 51:199203,2008

21. Kalavakonda C, Sekhar LN, Ramachandran P, Hechl P: Endoscope-assisted microsurgery for intracranial aneurysms. Neurosurgery 51:1119-1127, 2002

22. Kallmes DF, Kallmes MH: Cost-effectiveness of angiography performed during surgery for ruptured intracranial aneurysms. AJNR Am J Neuroradiol 18:1453-1462, 1997

23. Kallmes DF, Kallmes MH, Lanzino G, Kassell NF, Jensen ME, Helm GA: Routine angiography after surgery for ruptured intracranial aneurysms: a cost versus benefit analysis. Neurosurgery 41:629-641, 1997

24. Kato Y, Sano H, Nagahisa S, Iwata S, Yoshida K, Yamamoto $\mathrm{K}$, et al: Endoscope-assisted microsurgery for cerebral aneurysms. Minim Invasive Neurosurg 43:91-97, 2000

25. Katz JM, Gologorsky Y, Tsiouris AJ, Wells-Roth D, Mascitelli J, Gobin YP, et al: Is routine intraoperative angiography in the surgical treatment of cerebral aneurysms justified? A consecutive series of 147 aneurysms. Neurosurgery 58:719-727, 2006

26. Klopfenstein JD, Spetzler RF, Kim LJ, Feiz-Erfan I, Han PP, Zabramski JM, et al: Comparison of routine and selective use of intraoperative angiography during aneurysm surgery: a prospective assessment. J Neurosurg 100:230-235, 2004

27. Macdonald RL, Wallace MC, Kestle JR: Role of angiography following aneurysm surgery. J Neurosurg 79:826-832, 1993

28. Mielke D, Malinova V, Rohde V: Comparison of intraoperative microscopic and endoscopic ICG angiography in aneurysm surgery. Neurosurgery 10 (Suppl 3):418-425, 2014

29. Murphy M, Bell D, Worth RD, Jehle KS, Critchley GR, Norris JS: Angiography postclipping and coiling of cerebral aneurysms. Br J Neurosurg 19:225-228, 2005

30. Nishiyama Y, Kinouchi H, Senbokuya N, Kato T, Kanemaru $\mathrm{K}$, Yoshioka H, et al: Endoscopic indocyanine green video angiography in aneurysm surgery: an innovative method for intraoperative assessment of blood flow in vasculature hidden from microscopic view. J Neurosurg 117:302-308, 2012

31. Perneczky A, Boecher-Schwarz HG: Endoscope-assisted 
microsurgery for cerebral aneurysms. Neurol Med Chir (Tokyo) 38 Suppl:33-34, 1998

32. Perneczky A, Fries G: Endoscope-assisted brain surgery: part 1-evolution, basic concept, and current technique. Neurosurgery 42:219-225, 1998

33. Raabe A, Beck J, Gerlach R, Zimmermann M, Seifert V: Near-infrared indocyanine green video angiography: a new method for intraoperative assessment of vascular flow. Neurosurgery 52:132-139, 2003

34. Raabe A, Beck J, Seifert V: Technique and image quality of intraoperative indocyanine green angiography during aneurysm surgery using surgical microscope integrated nearinfrared video technology. Zentralbl Neurochir 66:1-8, 2005

35. Raabe A, Nakaji P, Beck J, Kim LJ, Hsu FP, Kamerman JD, et al: Prospective evaluation of surgical microscopeintegrated intraoperative near-infrared indocyanine green videoangiography during aneurysm surgery. J Neurosurg 103:982-989, 2005

36. Rauzzino MJ, Quinn CM, Fisher WS III: Angiography after aneurysm surgery: indications for "selective" angiography. Surg Neurol 49:32-41, 1998

37. Sindou M, Acevedo JC, Turjman F: Aneurysmal remnants after microsurgical clipping: classification and results from a prospective angiographic study (in a consecutive series of 305 operated intracranial aneurysms). Acta Neurochir (Wien) 140:1153-1159, 1998

38. Smith TR, Cote DJ, Dasenbrock HH, Hamade YJ, Zammar SG, El Tecle NE, et al: Comparison of the efficacy and safety of endovascular coiling versus microsurgical clipping for unruptured middle cerebral artery aneurysms: a systematic review and meta-analysis. World Neurosurg 84:942-953, 2015
39. Tang G, Cawley CM, Dion JE, Barrow DL: Intraoperative angiography during aneurysm surgery: a prospective evaluation of efficacy. J Neurosurg 96:993-999, 2002

\section{Disclosures}

Joachim Oertel is a consultant with Karl Storz Company.

\section{Author Contributions}

Conception and design: all authors. Acquisition of data: all authors. Analysis and interpretation of data: all authors. Drafting the article: all authors. Critically revising the article: Oertel, Fischer.

\section{Supplemental Information \\ Videos \\ Video 1. https://vimeo.com/275081946. \\ Video 2. https://vimeo.com/275081982.}

\section{Previous Presentations}

Portions of this work were presented in abstract form at the 65th Annual Meeting of the German Society of Neurosurgery, Dresden, Germany, May 11-14, 2014, and at the 66th Annual Meeting of the German Society of Neurosurgery, Karlsruhe, Germany, June 7-10, 2015.

\section{Correspondence}

Joachim Oertel: Universität des Saarlandes, Homburg, Germany. oertelj@freenet.de. 